\title{
THE LAW OF INFINITE CARDINAL ADDITION IS WEAKER THAN THE AXIOM OF CHOICE
}

\author{
BY
}

\author{
J. D. HALPERN AND PAUL E. HOWARD
}

Dedicated to the memory of Andrzej Mostowski

\begin{abstract}
We construct a permutation model of set theory with urelements in which $C_{2}$ (the choice principle restricted to families whose elements are unordered pairs) is false but the principle, "For every infinite cardinal $m, 2 m=m$ " is true. This answers in the negative a question of Tarski posed in 1924. We note in passing that the choice principle restricted to well-ordered families of finite sets is also true in the model.
\end{abstract}

1. The axiom of choice $(A C)$ implies that cardinal arithmetic is trivial for infinite cardinals. That is:

$$
m+n=\sup (m, n) \text { if one of } m, n \text { is infinite }
$$

and

$m \cdot n=\max (m, n)$ if one of $m, n$ is infinite and the other is different from 0.

Of course $A C$ implies that any two cardinals are comparable so $\sup (m, n)=$ $\max (m, n)$, but each of the laws as stated above follow respectively from the following laws without additional appeal to $A C$.

(1) $m+m=m$ for all infinite cardinals $m$.

(2) $m \cdot m=m$ for all infinite cardinals $m$.

In 1924 Tarski [3] gave a proof of $(A C)$ from (2) and asked [4] if $(A C)$ is provable from (1). We give a negative answer to this question by exhibiting a permutation model of $Z F U$ (Zermelo-Fraenkel set theory modified so as to allow urelements) in which $C_{2}$ (the axiom of choice for families whose elements are pairs) is false but (1) is true.(1)

Presented to the Society, January 15, 1974 under the title Cardinal addition and the axiom of choice; received by the editors February 1, 1975.

AMS (MOS) subject classifications (1970). Primary 02 K05.

(1) The proof of our result was completed near the end of December, 1972. We learned subsequently that G. Sageev (Notices Amer. Math. Soc. 20 (1973), A22) had already shown that the answer to Tarski's question was negative even in the context of set theory with regularity.

This paper was presented to the Association for Symbolic Logic in January 1973, to the Michigan-Ohio Logic Seminar in May and June 1973, to the International Colloquium in Infinite and Finite Sets at Keszthely, Hungary in June 1973. It was also presented as a Research Announcement in Bull. Amer. Math. Soc. 80 (1974), under a different title [1]. 
A permutation model (Specker [2]) is determined by a set of points(2) (i.e. urelements) $U$, a group $G$ of permutations of $U$, and a conjugated filter $J$ of subgroups of $G$.

Let $U$ be a countable set of points. $G$ and $J$ will be described in terms of an arbitrary 1-1 correspondence between $U$ and $\omega^{(\omega)}$ where $\omega^{(\omega)}$ is the set of all $s \in{ }^{\omega} \omega$ which are eventually zero, i.e., $(\exists n)(\forall j>n) s_{j}=0$. For ease of exposition, in the sequel we will not distinguish between $s \in \omega^{(\omega)}$ and the element in $U$ to which it corresponds. For $s \in U$, let the pseudo-length of $s$ be defined as the least number $k$ such that ${ }^{\circ} \forall l \geqslant k, s_{l}=0$. Call a permutation $\varphi$ of $U$ bounded if the set of those elements moved by $\varphi$ is of bounded pseudo-length i.e. for some $k, \varphi(s) \neq s$ implies the pseudo-length of $s$ is less than $k$. Let $G$ be the group of all bounded permutations of $U$. For $n \in \omega, s \in U$ let $n \mid s$ (read "s after $n$ ") $=\left(s_{n}, s_{n+1}, \ldots\right.$ ) and let $A_{s}^{n}$ (read "the $n$-block containing s") $=\{t \in U: n|t=n| s\}$ except that we will let $A^{n}$ be the $n$-block containing 0 , the sequence which is identically zero. Note the following: (1) If $s$ has pseudo-length $n$, then $n \mid s=0$. (2) $\varphi \in G$ implies that for some $k$, the set of those points moved by $\varphi$ (this set is usually called the support of $\varphi$ but we will be using "support" in a different way) is included in $A^{k}$. Also note that $s \in U$ is uniquely determined (for each $n \in \omega$ ) by $s \mid n$ and $n \mid s$. We will call $s \mid n$ the $n$-location of $s$. For each $n \in \omega$ let $G_{n}=\{\varphi \in G: 1$ and 2 and 3$\}$ where

1. $\varphi$ fixes the $n$-block containing 0 , i.e., $A^{n}$, pointwise.

2. $\varphi$ fixes $\left\{A_{s}^{n}: s \in U\right\}$, i.e., $n|s=n| t$ implies $n|\varphi(s)=n| \varphi(t)$.

3. $\varphi$ preserves $n$-locations, i.e., $s|n=\varphi(s)| n$.

Note that $G_{0}=\{\varphi \in G: \varphi(0)=0\}$. Also note that if $f$ is a 1-1 partial function on $U$ into $U$, having an extension in $G$ satisfying 1,2 , and 3 then $f$ has an extension in $G_{n}$. Let $J=$ the filter of subgroups generated by the $G_{n}, n \in \omega$.

LEMMA 1.1. $J$ is a proper filter and closed under conjugation.

Proof. $J$ is proper since $n<m$ implies $\left\{1_{G}\right\} \neq G_{m} \subseteq G_{n} . J$ is closed under conjugation since if $\{a: \varphi(a) \neq a\}$ is included in $A^{k}$ (and $k \geqslant n$ ) then $G_{k} \subseteq \varphi^{-1} G_{n} \varphi$.

Briefly the description of $M$ is as follows: Let $R$ be the function on ordinals defined recursively by $R(0)=U$ and $R(\alpha)=P\left(U_{\beta<\alpha} R_{\beta}\right)$ for $\alpha>0$. Then $V=\bigcup_{\alpha \in \text { ord }} R(\alpha)$ is called the set universe over $U$. Any permutation $\varphi$ of $U$ extends uniquely to an automorphism (with respect to $\Theta$ ) $\varphi^{*}$ of $V$.

(2) Permutation models can also be used for set theories whose axioms are those of $Z F$ except that the axiom of regularity is weakened or eliminated. Specker deals with such a theory but his development of permutation models carries over verbatim for $Z F U$. We use $Z F U$ rather than such a theory because it seems more natural. 
We will confuse $\varphi^{*}$ with $\varphi$ in the future. In the style of Lévy call an $x \in V$, $J$-symmetric, if there exists $H \in J$ such that $H$ fixes $x$, i.e., $\varphi \in H \Rightarrow \varphi(x)=x$. Then $M$ is just the substructure of $V$ consisting of all those sets $x$ such that $x$ and every element of the transitive closure of $x$ is $J$-symmetric.

Lemma 1.2. If $A \subseteq U$ and $A \in M$ then $M \vDash$ ( $A$ is countable) (i.e. $A$ is $M$ countable) iff $\exists k \in \omega$, such that $A \subseteq A^{k}$.

Proof. If $M \vDash$ ( $A$ is countable), then some $G_{k}$ fixes a $1-1$ correspondence between $A$ and $\omega$. Hence $G_{k}$ fixes $A$ pointwise and therefore $A \subseteq A^{k}$.

If $A \subseteq A^{k}$, then any 1-1 correspondence between $A$ and $\omega$ is fixed by $G_{k}$. Hence $A$ is $M$-countable.

THEOREM 1. $C_{2}$ is false in $M$.

Proof. The set of all (unordered) pairs of elements of $U$ is in $M$. However for any $k$ there is a $\varphi \in G_{k}$ such that $\varphi$ interchanges two elements of $U$. Hence there is no $J$-symmetric choice set for this set of pairs.

It remains to show that (1) holds in the structure $M$. To this end consider a set $y \in M$ and take $k_{0}$ such that $G_{k_{0}-1}$ fixes $y$ and $k_{0}>0$. Let:

$y_{1}=\left\{x \in y: G_{k_{0}}\right.$ fixes $\left.x\right\}$,

$y_{2}=\left\{x \in y: G_{k_{0}}\right.$ does not fix $x$ and there exists an $M$-countable $A \subseteq U$ such that $\forall \varphi \in G_{k_{0}}(\varphi$ fixes $A$ pointwise implies $\varphi(x)=x)$ \},

$y_{3}=\left\{x \in y:\right.$ for all $M$-countable $A \subseteq U, \exists \varphi \in G_{k_{0}}$ such that $\varphi$ fixes $A$ pointwise and $\varphi(x) \neq x$ \}.

Then $y$ is the disjoint union of $y_{1}, y_{2}$, and $y_{3}$. Furthermore $y_{1}, y_{2}, y_{3}$ $\in M$ since $G_{k_{0}}$ fixes each of them. (Actually $G_{k_{0}-1}$ fixes $y_{3}$. We will use this fact in §3.) $y_{1}$ is well orderable in $M$ since $G_{k_{0}}$ fixes each element and hence a well ordering of $y_{1}$. We will show that for $z=y_{2}$ or $z=y_{3}$ there is a 1-1 function $F$ with domain $2 \times z$ and range $z$ such that $G_{k_{0}+1}$ fixes $F$ and hence $F \in M$. We will call such an $F$ a $2 m$-ing of $z$. This shows that every cardinal in $M$ is the sum of three cardinals one of which is well orderable and the other two satisfy the equation $2 m=m$ and hence that (1) is true in the structure $M$.

(Note. If $y$ is finite, it follows that $y_{2}$ and $y_{3}$ must be empty and from this it follows that any well-ordered collection of finite sets in $M$ has a choice function in $M$.)

2. $y_{2}$ is $2 m$-able.

Definition 2.1. An $M$-countable $A \subseteq U$ is called an $n$-support of $x \in M$ if

(a) $\forall \varphi \in G_{n}, \varphi$ fixes $A$ pointwise $\Rightarrow \varphi(x)=x$.

(b) $\forall s \in U, A_{s}^{n} \cap A=\varnothing$ or $A_{s}^{n} \subseteq A$.

(c) $A^{n} \subseteq A$.

((a) is the crucial property; (b) and (c) are added for ease of exposition.) 
LEMMA 2.2. There are two functions

such that

$$
f_{0}, f_{1}: U-A^{k_{0}} \underset{\text { into }}{\stackrel{1-1}{\longrightarrow}} U-A^{k_{0}}
$$

(a) $f_{0}, f_{1}$ have disjoint ranges.

(b) $f_{0}, f_{1}$ are each fixed by $G_{k_{0}+1}$ and thus $f_{0}, f_{1} \in M$.

(c) $f_{0}, f_{1}$ preserve $k_{0}$-locations and each maps $k_{0}$-blocks to $k_{0}$-blocks; so the restrictions of $f_{0}, f_{1}$ to an $M$-countable set have extensions in $G_{k_{0}}$.

Proof. Let $f_{0}(s)=s^{\prime}$ where $s^{\prime}$ is like $s$ except that $s_{k_{0}}^{\prime}=2 s_{k_{0}}$. Let $f_{1}(s)=s^{\prime \prime}$ where $s^{\prime \prime}$ is like $s$ except that

It is clear that

$$
\begin{array}{ll}
s_{k_{0}}^{\prime \prime}=2 s_{k_{0}}+1 & \text { if } s \notin A^{k_{0}+1} \\
s_{k_{0}}^{\prime \prime}=2 s_{k_{0}}-1 & \text { if } s \in A^{k_{0}+1}-A^{k_{0}} .
\end{array}
$$

$$
f_{0}, f_{1}: U-A^{k_{0}} \underset{\text { into }}{\stackrel{1-1}{\longrightarrow}} U-A^{k_{0}} \text {. }
$$

Parts (a) and (c) are also clear. We prove that $G_{k_{0}+1}$ fixes $f_{0}$. The proof that $G_{k_{0}+1}$ fixes $f_{1}$ is almost identical.

Suppose $\varphi \in G_{k_{0}+1}$, then

$$
\phi\left(\left\langle s, f_{0}(s)\right\rangle\right)=\left\langle\phi(s), \phi\left(f_{0}(s)\right)\right\rangle
$$

We now compute

$\left[\phi\left(f_{0}(s)\right)\right]_{i}=\left\{\begin{array}{ll}\phi(s)_{i} & \text { if } i>k_{0} \text { since } \phi \in G_{k_{0}+1} \text { and }\left(k_{0}+1\right)\left|s=\left(k_{0}+1\right)\right| f_{0}(s), \\ 2 s_{i} & \text { if } i=k_{0} \\ s_{i} & \text { if } i<k_{0}\end{array}\right\}$ since $\phi \in G_{k_{0}+1}$ and

$$
\left[f_{0}(\phi(s))\right]_{i}= \begin{cases}\phi(s)_{i} & \text { if } i>k_{0} \\ 2[\phi(s)]_{i}=2 s_{i} & \text { if } i=k_{0} \\ \phi(s)_{i}=s_{i} & \text { if } i<k_{0}\end{cases}
$$

Therefore $\varphi\left(f_{0}(s)\right)=f_{0}(\varphi(s))$. So rewriting (*)

$$
\phi\left(\left\langle s, f_{0}(s)\right\rangle\right)=\left\langle\phi(s), f_{0}(\phi(s))\right\rangle \in f_{0} .
$$

Since $G_{k_{0}+1}$ is a group the above computation also holds for $\varphi^{-1}$. Hence $\varphi$ fixes $f_{0}$.

LEMMA 2.3 (SUPPORT LEMMA). If $A$ is a $k_{0}$-support of $x$, and $\varphi_{1}, \varphi_{2} \in$ $G_{k_{0}}$ and $\varphi_{1}\left|A=\varphi_{2}\right| A$, then $\varphi_{1}(x)=\varphi_{2}(x)$. 
This lemma follows from the facts that $\varphi_{2}^{-1} \varphi_{1} \in G_{k_{0}}$ and $\varphi_{2}^{-1} \varphi_{1}$ fixes $A$ pointwise.

Suppose $A$ is a $k_{0}$-support of $x \in y_{2}$. Then $f_{0} \mid A,\left(f_{1} \mid A\right)$, has an extension in $G_{k_{0}}$. The extension is not unique but the following corollary which is a direct consequence of the support lemma gives the desired amount of uniqueness.

Corollary 2.3. If $\varphi$ and $\varphi^{\prime}$ both extend $f_{0} \mid A,\left(f_{1} \mid A\right)$, where $A$ is a $k_{0}$-support of $x$ and $\varphi, \varphi^{\prime} \in G_{k_{0}}$, then $\varphi(x)=\varphi^{\prime}(x)$.

Lemma 2.4. If $A$ and $A^{\prime}$ are $k_{0}$-supports of $x$ then so is $A \cap A^{\prime}$.

Proof. We need only show that if $\varphi \in G_{k_{0}}$ and $\varphi$ fixes $A \cap A^{\prime}$ pointwise then $\varphi(x)=x$. We show that $\varphi$ can be represented as a finite sequence of elements of $G_{k_{0}}$ such that each element of the sequence fixes $A$ pointwise or fixes $A^{\prime}$ pointwise. Essentially such a representation is possible because $A$ and $A^{\prime}$ consist of $k_{0}$-blocks and $G_{k_{0}}$ is $M$-countably transitive on the set of $k_{0}$-blocks.

Choose an integer $m$ such that $m>k_{0}+1$ and $A^{m}$ has the following properties:

1. $\left(\forall s \in U-A^{m}\right)(\varphi(s)=s)$,

2. $A \cup A^{\prime} \subseteq A^{m}$. $m$ exists because $\varphi$ is bounded, $A$ and $A^{\prime}$ are supports, and for $m \leqslant n, A^{m} \subseteq A^{n}$. Let $B$ be any $m$-block different from $A^{m}$. (For definiteness one might take $B=A_{t}^{m}$ where $t$ is the sequence defined by $t_{m}=1$ and $t_{j}=0$ for $j \neq m$.) Let $\eta_{1}$ be the element of $\bar{G}_{k_{0}}$ which interchanges each element $s \in A^{\prime}-A$ with the element $r \in B$ having the same $m$-location as $s$ (i.e. $s|m=r| m$ ). $\eta_{1}$ is the identity elsewhere. Let $\eta_{2}$ be the element of $G_{k_{0}}$ which interchanges each element $s \in A^{m}-A^{\prime}$ with the element $r \in B$ having the same $m$-location as $s$.

$\eta_{2}$ is the identity elsewhere. Let $\varphi_{1}$ be the element of $G_{k_{0}}$ which is identical to $\varphi$ except that $\varphi_{1}$ acts on $B$ instead of $A^{m}$, that is $\varphi_{1}=\left(\eta_{2} \eta_{1}\right) \varphi\left(\eta_{2} \eta_{1}\right)$. Then $\eta_{1}$ fixes $A$ pointwise, $\eta_{2}$ fixes $A^{\prime}$ pointwise and $\varphi_{1}$ fixes $A \cup A^{\prime}$ pointwise. Clearly $\varphi=\eta_{1} \eta_{2} \varphi_{1} \eta_{1} \eta_{2}$. Hence the proof is complete. $A^{k_{0}}$.

COROLlaRY 2.5. If $A$ and $A^{\prime}$ are $k_{0}$-supports of $x \in y_{2}$ then $A \cap A^{\prime} \nsubseteq$

Proof. If $A \cap A^{\prime} \subseteq A^{k_{0}}$, then $A^{k_{0}}$ is a support of $x$ by Lemma 2.4 and hence $\varphi \in G_{k_{0}} \Rightarrow \varphi(x)=x$ contradicting $x \in y_{2}$. The following lemma is a direct consequence of 2.3 and 2.4 .

LEMMA 2.6. If $A$ and $A^{\prime}$ are $k_{0}$-supports of $k, \varphi \supseteq f_{0}\left|A, \varphi^{\prime} \supseteq f_{0}\right| A^{\prime}$ and $\varphi, \varphi^{\prime} \in G_{k_{0}}$ then $\varphi(x)=\varphi^{\prime}(x)$. (Similarly for $f_{1}$.)

DEFINITION 2.7. For each $x \in y_{2}$ let $x_{0},\left(x_{1}\right)$, be the unique member of 
$\left\{\varphi(x): \exists A, A\right.$ is a $k_{0}$-support of $x, \varphi \in G_{k_{0}}$ and $\left.\varphi \supseteq f_{0} \mid A,\left(\varphi \supseteq f_{1} \mid A\right)\right\}$.

Note. $x_{0}, x_{1} \in y_{2}$ since $G_{k_{0}}$ fixes $y_{2}$.

THEOREM 2. The function $F$ defined by $F(0, x)=x_{0}, F(1, x)=x_{1}$ is $a$ 1-1 function from $2 \times y_{2}$ into $y_{2}$ and is in $M$ (fixed by $G_{k_{0}+1}$ ).

Proof. $F \in M$ since the group $G \in M$ and hence the description of $x_{0}$ and $x_{1}$ in terms of $x$ can be carried out in $M$. This uses the fact that $M$ is a model of $Z F U$. It remains to prove that $F$ is 1-1. Let $x, z \in y_{2}$ and let $A$ be a $k_{0}$-support of both $x$ and $z$. (The union of a $k_{0}$-support of $x$ with a $k_{0}$-support of $z$ is a $k_{0}$-support of both.) Let $\varphi, \varphi_{1} \in G_{k_{0}}$ such that $\varphi \supseteq f_{0} \mid A$ and $\varphi_{1} \supseteq f_{1} \mid A$. Since $\varphi$ and $\varphi_{1}$ are automorphisms of $M$ we have $x \neq z \Rightarrow \varphi(x) \neq$ $\varphi(z)$ and $\varphi_{1}(x) \neq \varphi_{1}(z)$. Hence $F(0, x) \neq F(0, z)$ and $F(1, x) \neq F(1, z)$. It remains to show that $F(0, x) \neq F(1, z)$ i.e. $x_{0} \neq z_{1}$. Well, $x_{0}$ and $z_{1} \in y_{2}$, $\varphi(A)$ is a support of $x_{0}$ and $\varphi_{1}(A)$ is a support of $z_{1}$. But $\varphi(A) \cap \varphi_{1}(A)=A^{k_{0}}$. So the equality of $x_{0}$ and $z_{1}$ contradicts Corollary 2.5. Q.E.D.

3. $y_{3}$ is $2 m$-able. We will prove the following theorem:

TheOREM 3. For every $x \in y_{3}$ there is an infinite set $D_{x} \in M$ such that:

(1) $D_{x} \subseteq y_{3}$,

(2) $\forall \varphi \in G_{k_{0}+1}, \forall z_{1}, z_{2} \in D_{x}\left[\varphi\left(z_{1}\right)=z_{1}\right.$ iff $\varphi\left(z_{2}\right)=z_{2}$ and $\left(z_{1} \neq z_{2}\right.$ $\left.\left.\Rightarrow \varphi\left(z_{1}\right) \neq z_{2}\right)\right]$,

(3) $x \in D_{x}$.

Before proving the theorem we show why the theorem is sufficient to obtain the $2 m$-ing of $y_{3}$ in $M$.

For each $x \in y_{3}$, let

$$
M_{x}=\left\{y: \forall \phi \in G_{k_{0}+1}, \phi(y)=y \text { iff } \phi(x)=x\right\}
$$

and let $D_{x}=\left\{\varphi(y): y \in M_{x} \& \varphi \in G_{k_{0}+1}\right\}$.

LEMMA 3.1. $\left\{D_{x}: x \in y_{3}\right\}$ is a partition of $y_{3}$ each element of which is invariant under $G_{k_{0}+1}$.

The proof is an easy calculation. It suffices to obtain for each $x$, a $2 m$-ing of $D_{x}$ invariant under $G_{k_{0}+1}$.

We define a transitivity class (relative to $G_{k_{0}+1}$ ) of $D_{x}$ to be a subset $z$ of $D_{x}$ such that for each $y \in z, z=\left\{\varphi(y): \varphi \in G_{k_{0}+1}\right\}$.

Let $x_{0} \in y_{3}$ and choose $D_{x_{0}} \subseteq M_{x_{0}}$ such that $D_{x_{0}}$ intersects each transitivity class of $D_{x_{0}}$ in one element. Any subset of $M_{x_{0}}$ is in the model $M$ since the element of $J$ which fixes $x_{0}$ also fixes each element of $M_{x_{0}}$. Hence $D_{x_{0}} \in M$. Then $\left\{\varphi\left(D_{x_{0}}\right): \varphi \in G_{k_{0}+1}\right\}$ is a partition of $D_{x_{0}} \cdot D_{x_{0}}$ is infinite by Theorem 3 
and well orderable in the model $M$ since it is a subset of $M_{x_{0}}$ which is well orderable in $M$. (Any well ordering of $M_{x_{0}}$ is in the model $M$.) Hence there is a $2 m$ ing, say $F$, of $D_{x_{0}}$ in the model $M$. Then for each $\varphi \in G_{k_{0}+1}, \varphi(F)$ is a $2 m$ ing of $\varphi\left(D_{x_{0}}\right)$ since $\varphi$ is an automorphism of the model $M$. Hence $\bigcup\{\varphi(F)$ : $\left.\varphi \in G_{k_{0}+1}\right\}$ is a $2 m$-ing of $D_{x_{0}}$, is in $M$, and is invariant under $G_{k_{0}+1}$.

DEFINITION 3.2. For any $\varphi \in G$, let $\operatorname{St}(\varphi)$ (read "the stand of $\varphi$ ") $=\{a \in$ $U: \varphi(a) \neq a\}$. ing lemma.

First we note that $G_{k_{0}-1}$ fixes $y_{3}$. This follows directly from the follow-

Lemma 3.3. For all $\varphi \in G, z \in M$, if $z$ has an $n$-support then $\varphi(z)$ has an n-support.

Proof. Let $B$ be an $n$-support of $z$ and let

$$
B^{\prime}=B \cup \bigcup\{A: A \text { is an } n \text {-block and } A \cap \operatorname{St}(\varphi) \neq 0\} \text {. }
$$

$B^{\prime}$ is $M$-countable since $\operatorname{St}(\varphi)$ is $M$-countable. Furthermore if $\psi \in G_{n}$ and leaves $B^{\prime}$ pointwise fixed then $\psi \varphi=\varphi \psi$. So $\psi \varphi(x)=\varphi(x)$. $B^{\prime}$ obviously satisfies the other requirements of an $n$-support of $\varphi(x)$.

Let $x \in y_{3}$ and let $k$ be the least number such that $x$ has $k$-support. $k>$ $k_{0}$ since $x \in y_{3}$.

Lemma 3.4. Let $B$ be a $k$-support of $x$. If $A$ is an M-countable subset of $U$, $A$ is the union of infinitely many $k$-blocks, and $A$ is disjoint from $B$, then there exists $\varphi \in G_{k-1}$ such that $\operatorname{St}(\varphi) \subseteq A$ and $\varphi(x) \neq x$.

Proof. Since $B$ is not a $(k-1)$-support of $x$, there exists $\varphi_{1} \in G_{k-1}$ such that $\varphi_{1}$ fixes $B$ pointwise and $\varphi_{1}(x) \neq x$. Let $A_{1}=\left\{C: C\right.$ is a $k$-block and $\varphi_{1}$ moves some element of $C$ \}. $\bigcup A_{1}$ is $M$-countable and disjoint from $B$, so there exists $\psi \in G_{k}$ such that $\psi$ interchanges $A_{1}$ with the set of $k$-blocks of $A$ (or a finite subset of the $k$-blocks of $A$ if $A_{1}$ is finite) and $\psi$ fixes $B$ pointwise. Then $\varphi=\psi \varphi_{1} \psi^{-1}$ has the desired properties.

DEFINITION 3.5. Let $\varphi$ be a 1-1 function with domain and range included in $U$. We say that $\varphi$ maps $n$-blocks to $k_{0}$-blocks uniformly if its domain is the union of $n$-blocks, its range is the union of $k_{0}$-blocks for any $s, s^{\prime} \in \operatorname{Domain}(\varphi)$

(i) $s \ln =s^{\prime} \ln$ implies $\varphi(s)\left|k_{0}=\varphi\left(s^{\prime}\right)\right| k_{0}$ and

(ii) $n|s=n| s^{\prime}$ implies $k_{0}\left|\varphi(s)=k_{0}\right| \varphi\left(s^{\prime}\right)$.

Note. If $\varphi$ maps $n$-blocks to $k_{0}$-blocks uniformly and $\psi \in G_{n}$ then $\varphi \psi \varphi^{-1}$ has an extension in $G_{k_{0}}$. Similarly, if $\psi_{1} \in G_{k_{0}}$, then $\varphi^{-1} \psi_{1} \varphi$ has an extension in $G_{n}$. Replacing "implies" by "iff" in the definition does not change its strength.

The following lemma is a consequence of the fact that $k,(k-1) \geqslant k_{0}$. 
LEMMA 3.6. If $B$ and $C$ are mutually disjoint $M$-countable subsets of $U-$ $A^{k_{0}-1}, B$ is the union of infinitely many $k$-blocks $((k-1)$-blocks), $C$ is the union of infinitely many $k_{0}$-blocks, then there exists

$$
\varphi: B \underset{\text { onto }}{\stackrel{1-1}{\longrightarrow}} C
$$

such that $\varphi$ maps $k$-blocks $\left((k-1)\right.$-blocks) to $k_{0}$-blocks uniformly, and $\varphi$ has an extension in $G_{k_{0}-1}$.

Proof. (We prove the lemma for $k$.) For each $n \in \omega$, let $A_{n}$ be the set of $n$-locations, i.e.

$$
A_{n}=\{s \mid n: n \in \omega\}=\{s: s \text { is a sequence of length } n\} .
$$

To prove the lemma it suffices to find a one-to-one function $g$ from $A_{k}$ onto $A_{k_{0}}$ with these two properties: For all $s, s^{\prime} \in A_{k}$

$$
s\left|k_{0}-1=g(s)\right| k_{0}-1
$$

and

$$
\left(k_{0}-1\right)\left|s=\left(k_{0}-1\right)\right| s^{\prime} \Rightarrow\left(k_{0}-1\right) \lg (s)=\left(k_{0}-1\right) \lg \left(s^{\prime}\right) .
$$

But $g$ can be defined by

$$
\begin{aligned}
& g\left(s_{0}, s_{1}, \ldots, s_{k_{0}-2}, s_{k_{0}-1}, \ldots, s_{k-1}\right) \\
& \quad=\left(s_{0}, s_{1}, \ldots, s_{k_{0}-2}, P_{k-k_{0}+1}\left(s_{k_{0}-1}, \ldots, s_{k-1}\right)\right)
\end{aligned}
$$

where $P_{k-k_{0}+1}$ is a $1-1$ function from $\omega^{k-k_{0}+1}$ onto $\omega$.

In the sequel, let $B$ be a fixed $k$-support of $x$, let $F$ be a $(k+1)$-block disjoint from $B$ and let $H=\left\{\varphi \in G_{k}: \varphi\right.$ is the identity on $\left.B\right\}$.

DEFINITION 3.7. Let

such that

$$
\delta_{1}: F \cup A^{k_{0}+1} \frac{1-1}{\text { onto }} A^{k_{0}+1}
$$

(i) $\delta_{1}$ has an extension in $G_{k_{0}-1}$,

(ii) $\delta_{1}$ maps $F 1-1$ and onto $A^{k_{0}+1}-A^{k_{0}}$,

(iii) $\delta_{1} \mid F$ maps $k$-blocks to $k_{0}$-blocks uniformly.

Let $\delta_{2}$ be a $1-1$ function from $U-\left(F \cup A^{k_{0}+1}\right)$ onto $U-A^{k_{0}+1}$ such that

(iv) $\delta_{2} \mid \mathrm{St}\left(\delta_{2}\right)$ has an extension in $G_{k}$,

(v) $\operatorname{St}\left(\delta_{2}\right)$ is the union of $k$-blocks and is disjoint from $B$.

Let $\Delta=\delta_{1} \cup \delta_{2}$. The existence of $\delta_{1}$ follows from Lemma 3.6. To get $\delta_{2}$, we note that $F \cap B=\varnothing$ and $A^{k} \subseteq B$, therefore $A^{k}-A^{k_{0}+1} \subseteq U-\left(F \cup A^{k_{0}+1}\right)$ ( = domain $\delta_{2}$ ). So $\delta_{2}$ can be (and in fact must be) chosen to be the identity on $A^{k}-A^{k_{0}{ }^{+1}}$. It then remains to define $\delta_{2}$ on $U-\left(F \cup A^{k}\right)$, but this set 
and $U-A^{k}$ are both unions of $k$-blocks and have $M$ countable complements so the existence of $\delta_{2}$ is clear. $\Delta$ is not uniquely determined by the definition but we assume it is fixed in the sequel.

Definition 3.8. Let $\sigma_{1}$ be a 1-1 function from $F \cup A^{k_{0}+1}$ onto $A^{k_{0}+1}$ such that

(i) $\sigma_{1}$ has an extension in $G_{k_{0}-1}$,

(ii) $\sigma_{1} \mid A^{k_{0}+1}$ has an extension in $G_{k_{0}}$,

(iii) $\sigma_{1} \mid F$ maps $(k-1)$-blocks to $k_{0}$-blocks uniformly.

Let $\sigma=\sigma_{1} \cup \sigma_{2}$. Again the existence of $\sigma_{1}$ follows from Lemma 3.6 mainly. $\sigma$ is not uniquely determined by the definition but we assume it is fixed in the sequel.

The main difference between $\sigma$ and $\Delta$ can be seen by considering the set $E=\left\{A_{s}^{k_{0}}: A_{s}^{k_{0}} \subseteq A^{k_{0}+1} \& s \neq 0\right\}$. Under $\Delta$ each element of $E$ is the image of some $k$-block which is a subset of $F$. On the other hand under $\sigma$ some elements of $E$ are images of $(k-1)$-blocks (which are subsets of $F$ ) while the remaining elements of $E$ are images of elements of $E$.

Note that $\sigma, \Delta \in G_{k_{0}-1}$. Let

$$
D=\left\{\Delta(x), \sigma \Delta(x), \ldots, \sigma^{(n)} \Delta(x), \ldots\right\}_{n \in \omega} .
$$

Then $D \subseteq y_{3}$.

LEMMA 3.9. For all $\varphi \in G$, if $\operatorname{St}(\varphi)$ is disjoint from $A^{k_{0}+1}$ then $\varphi \Delta(x)$ $\neq \sigma^{(n)} \Delta(x)$ for all $n \geqslant 1$.

Proof. Lemma 3.4 and Definition 3.7 (iv) and (v) assure the existence of $\psi \in G_{k-1}$ such that $\operatorname{St}(\psi) \subseteq \Delta^{-1}(F)$ and $\psi(x) \neq x$. Let $\Omega=\left(\sigma^{(n)} \Delta\right) \psi\left(\sigma^{(n)} \Delta\right)^{-1}$. Then $\Omega \sigma^{(n)} \Delta(x) \neq \sigma^{(n)} \Delta(x)$. On the other hand, $\operatorname{St}(\Omega) \subseteq A^{k_{0}+1}$ and 3.7 (iv) and (v) together with 3.8 (ii) and (iii) assure that $\Omega \in G_{k_{0}}$. This fact together with 3.7 (ii) and (iii) assures that $\Delta^{-1} \Omega \Delta \in H$. Hence $\Delta^{-1} \varphi^{-1} \Omega \varphi \Delta \in H$, i.e. $\Omega \varphi \Delta(x)=$ $\varphi \Delta(x)$. Thus $\Omega$ distinguishes $\varphi \Delta(x)$ from $\sigma^{(n)} \Delta(x)$.

COROLlaRY 3.10. If $n \neq m, \sigma^{(n)} \Delta(x) \neq \sigma^{(m)} \Delta(x)$. Thus $D$ is infinite.

COROLLARY 3.11. If $G_{k_{0}+1}$ and $n<m$ then $\varphi \sigma^{(n)} \Delta(x) \neq \sigma^{(m)} \Delta(x)$.

Proof. St $\left(\sigma^{(-n)} \varphi \sigma^{(n)}\right)$ is disjoint from $A^{k_{0}+1}$.

LEMMA 3.12. Let $\varphi \in G_{k_{0}+1}, n \in \omega$. Then $\varphi(x)=x$ iff $\varphi\left(\sigma^{(n)} \Delta(x)\right)=$ $\sigma^{(n)} \Delta(x)$.

Proof. It follows mainly from Definition 3.7 (iv) and (v) that there is $\psi \in H$ such that $\operatorname{St}(\varphi)$ is disjoint from $\operatorname{St}\left(\psi^{-1} \sigma^{(n)} \Delta \psi\right)$. Furthermore $\psi^{-1} \sigma^{n} \Delta \psi(x)=\psi^{-1} \sigma^{n} \Delta(x)$ since $\psi \in H$. Also $\left(\sigma^{n} \Delta\right)^{-1} \psi^{-1} \sigma^{n} \Delta \in H$ because of 
Definition 3.7 (iv) and (v). Thus $\psi^{-1} \sigma^{(n)} \Delta \psi(x)=\sigma^{(n)} \Delta(x)$, and $\varphi$ commutes with $\psi^{-1} \sigma^{(n)} \Delta \psi$. This is sufficient to give the desired result.

Proof of Theorem 3. It follows from 3.9 thru 3.12 that $D$ has all of the properties desired of $D_{x}$ except, perhaps, the property of containing $x$ as a member. If there is a $\varphi \in G_{k_{0}+1}$ such that $\varphi(x) \in D$, let $D_{x}=\varphi^{-1}(D)$. Otherwise let $D_{x}=D \cup\{x\}$. In either case the crucial properties of $D$ carry over to $D_{x}$.

\section{REFERENCES}

1. J. D. Halpern and Paul E. Howard, Cardinal addition and the axiom of choice, Bull. Amer. Math. Soc. 80 (1974), 584-586. MR 48 \#8230.

2. Ernst Specker, Zur Axiomatic der Mengenlehre (Fundierungs- und Auswahlaxiom), Z. Math. Logik Grundlagen Math. 3 (1957), 173-210. MR 20 \#5738.

3. Alfred Tajtelbaum-Tarski, Sur quelques théorèmes qui équivalent 'a l'axiome du choix, Fund. Math. 5 (1924), 147-154.

4. - Probleme 31, Fund. Math. 5 (1924), 338.

DEPARTMENT OF MATHEMATICS, UNIVERSITY OF ALABAMA-BIRMINGHAM, BIRMINGHAM, ALABAMA 35294

DEPARTMENT OF MATHEMATICS, EASTERN MICHIGAN UNIVERSITY, YPSILANTI, MICHIGAN 48197 